ISSN 1991- 8690

Website: http://jsci.utq.edu.iq

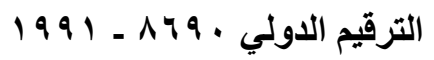

Email: utjsci@utq.edu.iq

\title{
Comparison the Performance of two Wireless Sensor Networks protocols (LEACH and EAMMH)
}

\author{
Rana Hameed Hussain ${ }^{1}$ \\ Ali Basim Yousif ${ }^{2}$ \\ ${ }^{1}$ Dep. of Computer Science College of computer sciences and mathematics \\ ${ }^{2}$ Dep. of Computer Science _College Of Education For Pure Sciences _Thi-Qar University
}

E-mail:Rana_hameed2003@yahoo.com

E-mail:alibasim8576@yahoo.com

\begin{abstract}
$\underline{\text { Abstract }}$
In Spite of advantages of using wireless sensor networks in many fields, lifetime of nodes and how energy consumption in each node it is one of the most important problems of this type of networks ,so developers of networks relied on the use of protocols helps reduce the energy consumption of nodes in the network. Therefore aims of this research is to compare the efficiency of the most popular protocols that used in wireless sensor network its (LEACH and EAMMH) has been adopted in several scenario and brief analysis of the simulation results against known metrics with energy and network lifetime being major among them to measure the efficiency of these two protocols. In this paper will the results and observations made from the analyses of results about these protocols are presented.
\end{abstract}

Keywords: Wireless sensor network, lifetime, LEACH, EAMMH, multi path.

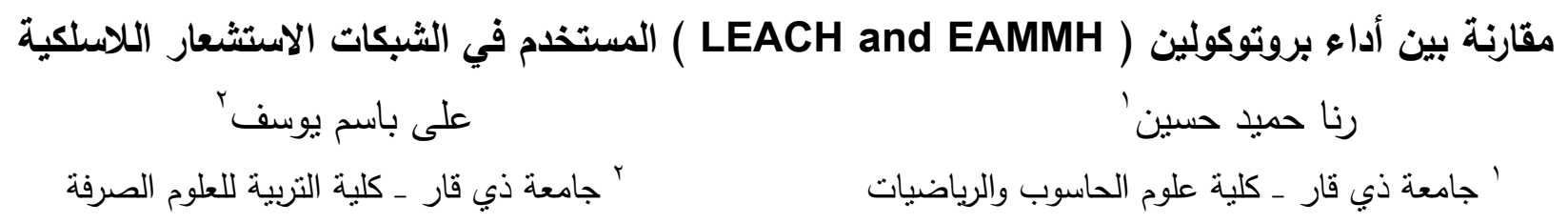

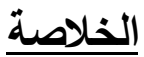

وعلى الرغم من المزايا العديدة لاستخدام شبكات الاستشعار اللاسلكية في الكثير المجالات، فان عمر العقد وكيفية استهلاك الطاقة في كل عقدة بعتبر

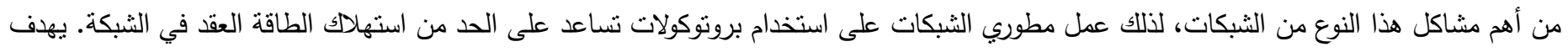

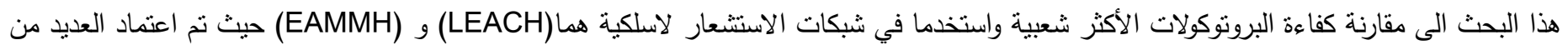
السيناريوات وتحليل موجز لنتائج المحاكاة لمقاييس الطاقة وعمر العقد داخل الثبكة معروفة لقياس كفاءة هذين البرونوكولين. في هذا البحث سوف نعرض وله

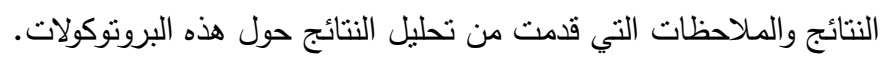
الكلمات المفتاحية: شبكات التحس اللاسلكية كبروتوكولات EAMMH،LEACH, وعمر العقدة ,المسارات المتعددة

\section{Introduction}

Recent advances in wireless communications and electronics have enabled the development of low-cost, low-power and multifunctional sensor nodes that are small in size and have sensing, data processing and communicating components. These devices leverage the idea of sensor networks [1].Power conservation and management is an important issue in Wireless Sensor Networks because the nodes are dependent on battery for their power. Sensors may be left unattended in hostile environments which makes it difficult or impossible to re-charge or replace their batteries 
especially when they deployed in large numbers. This necessitates devising novel energy-efficient solutions such as medium access control and routing. Network lifetime can be defined as the time elapsed until the first (or the last) node in the network runs out of energy(dies). The limited energy resource in the nodes of the wireless sensor networks puts a significant constraints on the power available for communications, thus affects both the transmission range and the data rate.Communication and computation processes should be bound to consume optimal power so it is highly desirable to find methods for energy-efficient routing and relaying of data from the sensor nodes to the base station so that the lifetime of the network is maximized [2].Researchers have proposed numerous routing protocols to improve performance of different application in a wireless sensor network. Most of the protocols in Wireless Sensor Networks are designed based on single path-routing strategy without considering the various effects of various load traffic intensities. A hop by hop basis data transfer increases the overhead on routing table management and quickly brings down the lifetime of those nodes which are near to the base station as these nodes will be used extensively as relay nodes. Such a network will be nonexistent as the energy of the nodes near the base station drains quickly. Many routing protocols have been suggested to overcome such issues $[3,4]$. Out of these, clustering algorithms have been of much interest as they well balance several key factors of Wireless Sensor Networks operation simultaneously [5]. Choosing one arbitrary node to act as servicing node for several sensor nodes than each trying to reach Gateway node can extend network lifetime and bring down energy utilization considerably. This process of choosing one node to act as servicing node for several neighbor nodes is known as 'clustering'. In this paper section are arranged as following: section 2 gives the brief working of LEACH protocol, section 3 also gives working of EAMMH protocol while section 4 presents details about simulation using MATLAB tool and the analysis of results is presented. finally in section 6 by mentioning the effectiveness of both LEACH and EAMMH.

\section{Low-Energy Adaptive Clustering Hierarchical (LEACH)}

LEACH is a self-organizing and adaptive clustering protocol [6]. It uses randomization to distribute the energy load evenly among the sensors in the network. In LEACH, the nodes organize themselves into local clusters, one of the cluster nodes acting as cluster-head while other nodes will serve as member nodes. In order to spread the energy usage over multiple nodes, LEACH suggests that the cluster-head nodes are not fixed. For example if ( $\mathrm{C}$ ) is the set of nodes might elect themselves as cluster-heads at time $\mathrm{t} 1$, at time $\mathrm{t} 1+$ $\mathrm{d}$, a new set ( $\mathrm{C}$ ) of nodes will select themselves as cluster-heads. The operation of LEACH is broken up into rounds, each round consists of two main phases; a set-up phase and a steady-state phase. In cluster setup phase, LEACH forms cluster in self-adaptive mode; in the second phase, it transfers data. Figure (1) shows the basic steps in LEACH algorithm.

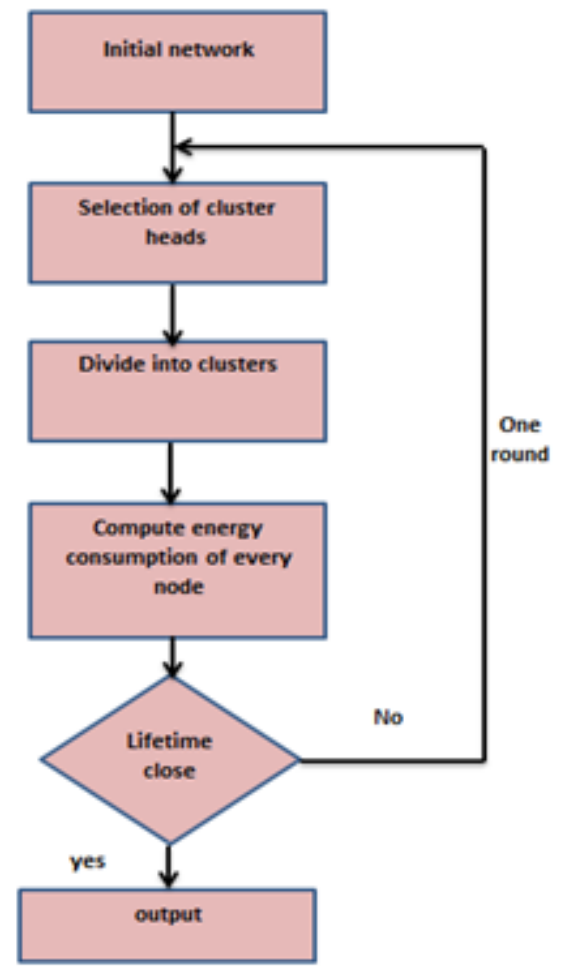

Figure (1):LEACH protocol

\subsection{Set-Up Phase}

It can be also subdivided into advertisement phase and cluster set-up phase, in advertisement phase each node decides, independently of other nodes, whether or not to be a cluster head for the current round. This decision takes into account when the node served as a $\mathrm{CH}$ for the last time and the priori determined percentage of the cluster heads for the network. Each node chooses a random number between 0 and 1 , if this number is less than a threshold $\mathrm{T}(\mathrm{n})$, then the node 
becomes a cluster head. $T(n)$ is calculated as in equation:

$$
T(n)=\left\{\begin{array}{lc}
\frac{p}{1-p\left(r \bmod \left(\frac{1}{p}\right)\right.} & \text { if } n \in G \\
0 & \text { otherwise }
\end{array}\right.
$$

Where $\mathrm{P}$ is the desired percentage of $\mathrm{CHs}, \mathrm{r}$ is the current round and $G$ is the set of nodes that have not been $\mathrm{CHs}$ in the last $(1 / \mathrm{p})$ rounds.

Nodes elected themselves as $\mathrm{CHs}$ broadcast "advertisement message" to the rest of the nodes and they use a CSMA MAC (Carrier Sense Multiple Access) protocol to transmit their advertisements. The non-CHs nodes must keep their receivers $\mathrm{ON}$ during this phase to hear the advertisements of all $\mathrm{CH}$ nodes, then each non- $\mathrm{CH}$ node decides the cluster to which it will belong for this round. This decision is based on the received signal strength of the advertisement, the cluster head with the largest signal strength is the $\mathrm{CH}$ to whom the minimum amount of transmitted energy is needed for communication. Cluster formation steps are explained in Figure (2). In the next cluster setup phase, each non- $\mathrm{CH}$ node informs its $\mathrm{CH}$ that it becomes one of its members using "join message" which contains its ID using CSMA. With this phase, each $\mathrm{CH}$ knows the number and the IDs of its members, then it can create a TDMA( Time Division Multiple Access) scheduling and broadcasts the TDMA table to cluster members.

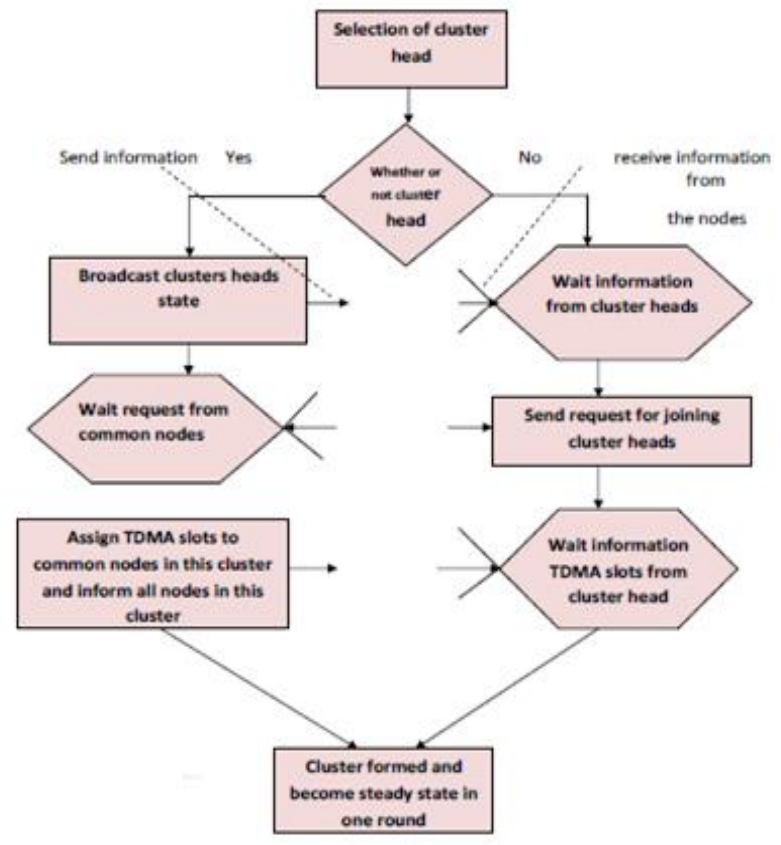

Figure (2): LEACH setup phase

\subsection{Steady-State Phase:}

It is data transmission phase, member nodes send their data during their allocated transmission time to the cluster head. The radio of each non-cluster head node can be turned off until the nodes allocated transmission time, thus minimizing energy dissipation in these nodes. The cluster-head node must keep its receiver $\mathrm{ON}$ to receive data from all its members, when all the data has been received, the cluster head aggregates these data and send it to the Base Station (BS) $[7,8]$.

\section{Energy Aware Multi-hop Multi-path Hierarchical (EAMMH) Routing Protocol \\ EAMMH routing protocol was developed by} inducing the features of energy aware routing and multi-hop intra cluster routing [9]. The operation of the EAMMH protocol is broken up into rounds where each round begins with a set-up phase, when the clusters are organized, followed by a steady- state phase, when data transfers to the base station occur. The below flow chart describes the overview of the protocol initially the user has to give the input which is in the form of number of nodes.For the nodes generated, their positions are randomly assigned and displayed. Once the nodes are deployed, every node uses the neighbor discovery algorithm to discover its neighbor nodes. Using the cluster head selection algorithm cluster heads are selected among the nodes. These cluster heads broadcasts the advertisement message to all its neighboring nodes and thus clusters are formed with a fixed bound size. Each node in the cluster maintains routing table in which routing information of the nodes are updated. distributed randomized time slot assignment algorithm (DRAND) method is used, it allows several nodes to share the same frequency channel by dividing the signal into different time slots. The cluster head aggregates the data from all the nodes in the cluster and this aggregated data is transmitted to the base station. 


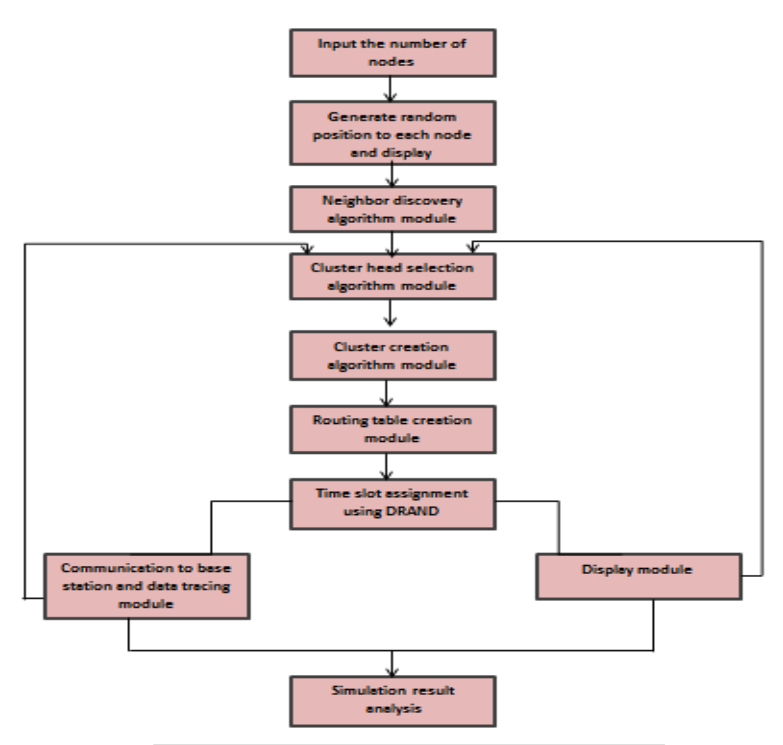

Figure (3): EAMMH protocol

\subsection{Setup Phase}

Initially, after the node deployment the neighbor discovery takes place. This can be done using many methods like: k-of-n approach, ping, beacon messaging. After the neighbor discovery [10], when cluster are being created, each node decides whether or not to become a cluster-head for the current round. This decision method is similar to the one used in LEACH. The setup phase operates in the following sequence:

1. CH (Cluster Head) Selection

2. Cluster Formation

\subsection{Data Transmission Phase}

Once the clusters are created, the sensor nodes are allotted timeslots to send the data. Assuming nodes always have data to send, they transmit it at their allotted time interval. When a node receives data from one its neighbors, it aggregates it with its own data. While forwarding the aggregated data, it has to choose an optimal path from its routing table entries. It uses a heuristic function to make this decision and the heuristic function is given by:

$$
\mathrm{h}=\mathrm{K}(\text { Eavg/ hmin * t ) (2) }
$$

where $\mathrm{K}$ is a constant, Eavg is average energy of the current path, hmin is minimum hop count in current path, $\mathrm{t}=$ traffic in the current path.

The path with highest heuristic value is chosen. If this path's Emin> threshold, it is chosen. Else the path with the next highest heuristic value is chosen, where

$$
\text { Emin }=\text { Eavg /const (3) }
$$

The constant may be any integer value like 10 .
If no node in the routing table has Emin greater than threshold energy, it picks the node with highest minimum energy.

\subsection{Periodic Updates}

The information about the paths and routing table entries at each node becomes stale after a little while. The heuristic values calculated based on the stale information often leads to wrong decisions. Hence the nodes are to be supplied with fresh information periodically. This will increase the accuracy and timeliness of the heuristic function. During the operation of each round, the necessary information is exchanged at regular intervals. The interval of periodic updates is chosen wisely such that the node does not base its decisions on the stale information and at the same time, the periodic update does not overload the network operation.

\section{Simulation And Analysis Of Results}

MATLAB was using to simulation both LEACH and EAMMH and the parameters taken into consideration are as follows:

1. Round Number vs. Number of Dead Nodes (with difference of probability)

2. Round Number vs. Average Energy of Each node (with difference of probability)

3. Round Number vs. Number of Dead Nodes (with difference of number of nodes)

4. Round Number vs. Average Energy of Each node (With difference of number of nodes)

We are taken few assumptions to simplify the simulation of these protocols they are (initial energy of nodes is same, nodes are static, nodes are assumed to have a limited transmission range after which a another equation for energy dissipation is used, homogeneous distribution of nodes, and nodes always have to send the data). And the details of the simulation environment are mentioned in Table 1.

Table 1: Simulation Details

\begin{tabular}{|c|c|}
\hline parameter & value \\
\hline Simulation Area & $100 * 100$ \\
\hline Base Station Location & $(150,50)$ \\
\hline Channel Type & Wireless Channel \\
\hline Energy Model & $10 * 0.000000000001$ \\
Transmission Amplifier & $0.0013 * 0.000000000001$ \\
Efs & $5 * 0.000000001$ \\
\hline Data Aggregation Energy & $50 * 0.000000001$ \\
\hline Transmission Energy,ETx & $50 * 0.000000001$ \\
Receiving Energy,ERX & \\
\hline \hline
\end{tabular}




\subsection{Simulation of protocols at 0.05 probability}

The below set of results represent the simulation of both LEACH and EAMMH protocols at 0.05 probability that is the percentage of total nodes which can become cluster head is $5 \%$ of the total number of nodes.

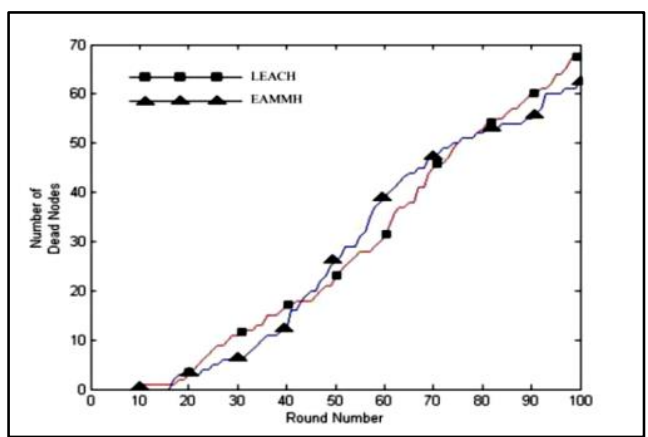

Figure (4):50 nodes

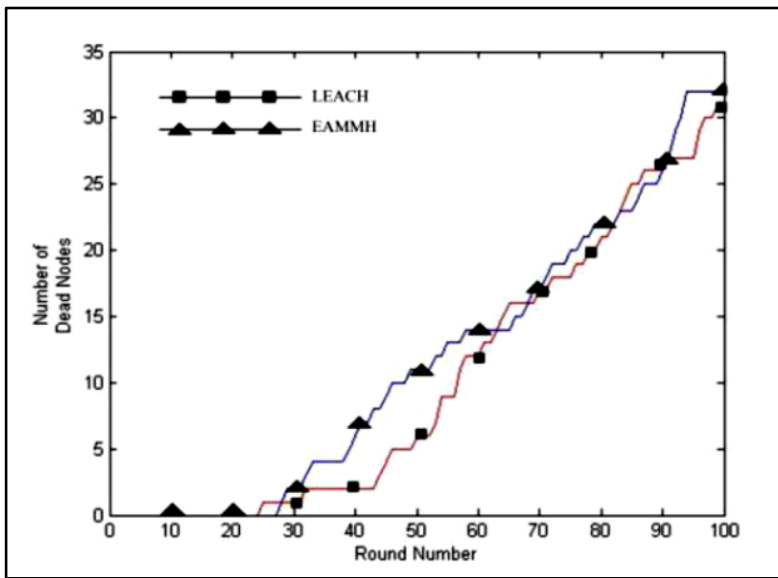

Figure (5):100 nodes

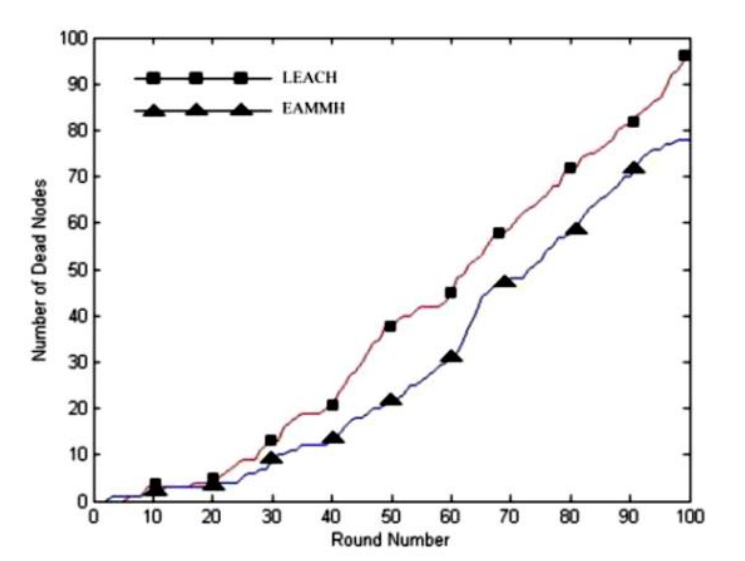

Figure (6):150 nodes

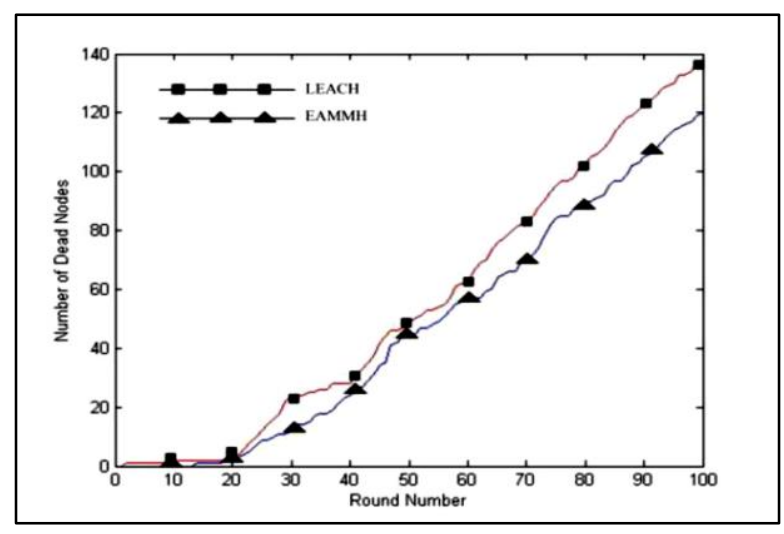

Figure (7):200 nodes

Figures 4, 5, 6 and 7 represent the comparison of LEACH and EAMMH protocols for the number of dead nodes against the round number elapsed for 50,100,150 and 200 nodes respectively. From Figure 4 we observe that, the number of dead nodes with the simulation of LEACH protocol is almost as comparable to number of dead nodes in EAMMH protocol. However as the number of nodes increase, we observe from Figure 5,6 and 7 that EAMMH results in lesser number of dead nodes after the completion of 100 rounds when compared to LEACH.

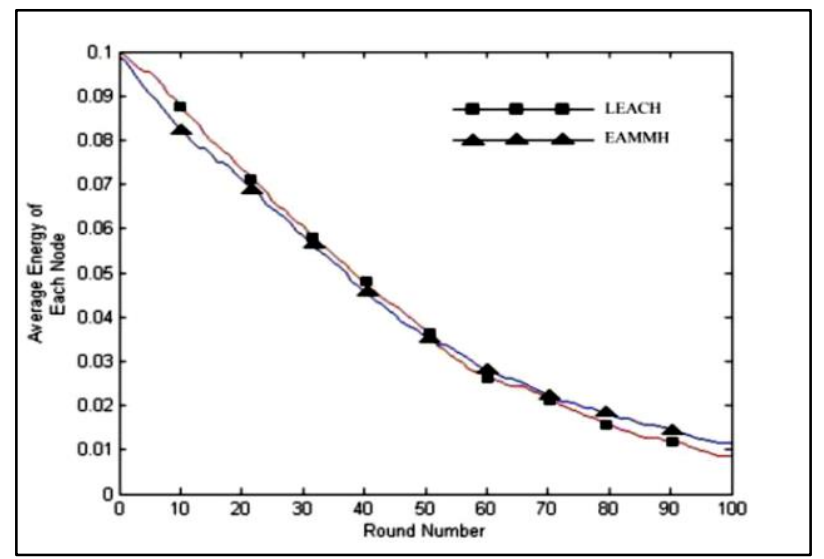

Figure (8):50 nodes 


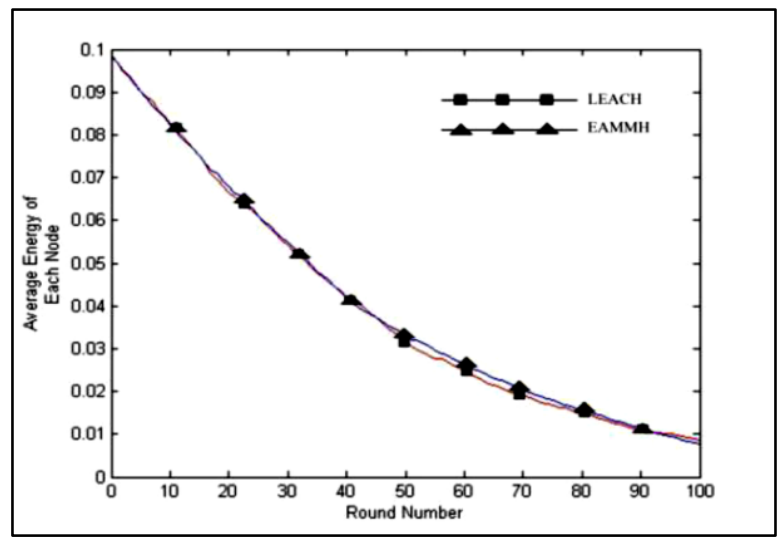

Figure (9):100 nodes

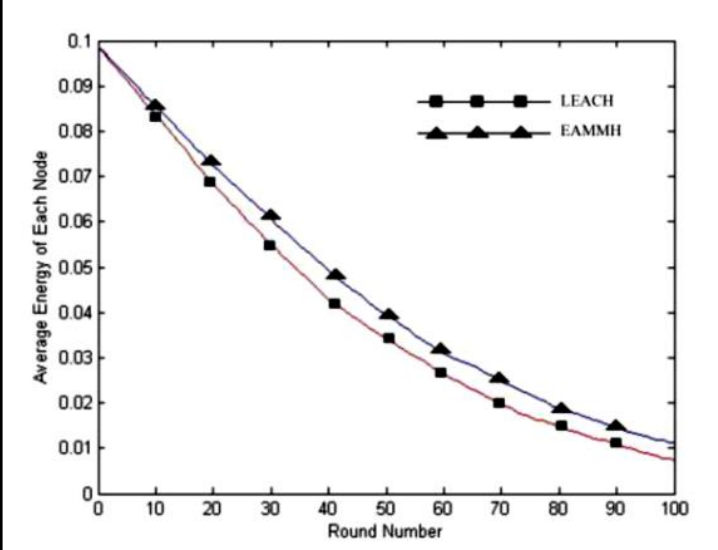

Figure (10):150 nodes

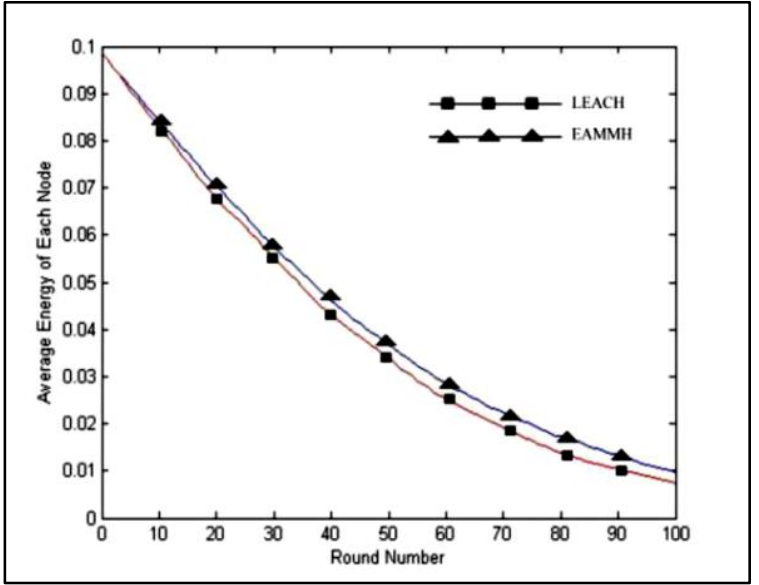

Figure (11):200 nodes
Figures 8,9,10 and 11 represents the average energy of each node as the round progresses for LEACH and EAMMH protocols. In Figure 8 and Figure 9, the average energy of each node after 100 rounds is almost equal for both EAMMH and LEACH whereas EAMMH performs slightly better in Figure 10 and Figure 11.

\subsection{Simulation of Protocols at 0.1 probability}

The previous set of results represent the simulation of both $\mathrm{LEACH}$ and EAMMH protocols at 0.1 probability that is the percentage of total nodes which can become cluster head is $10 \%$ of the total number of nodes.

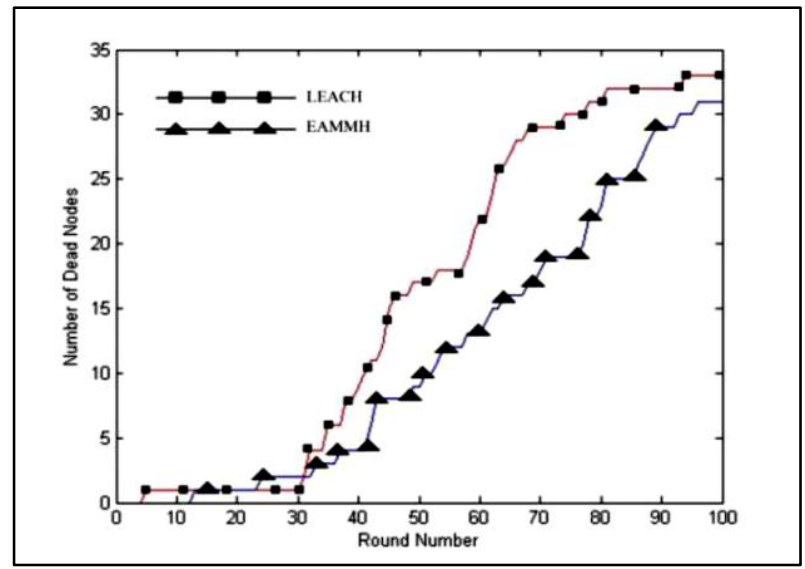

Figure (12):50 nodes

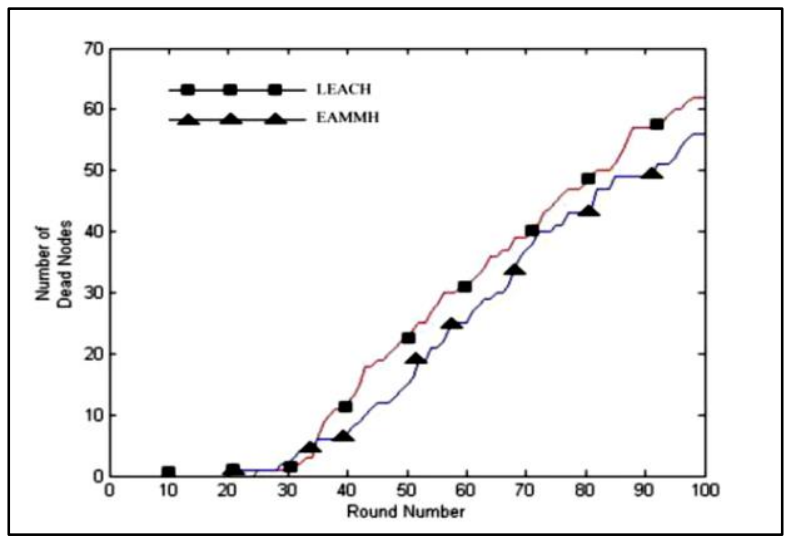

Figure (13):100 nodes 


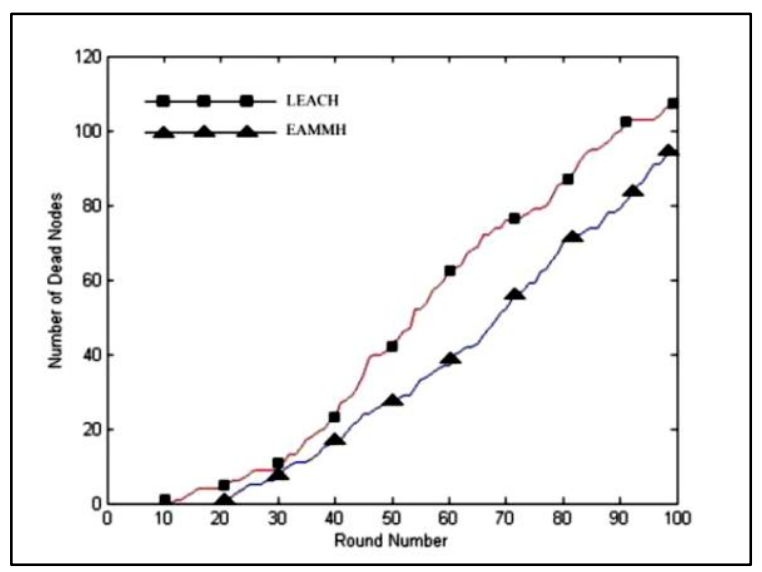

Figure (14):150 nodes

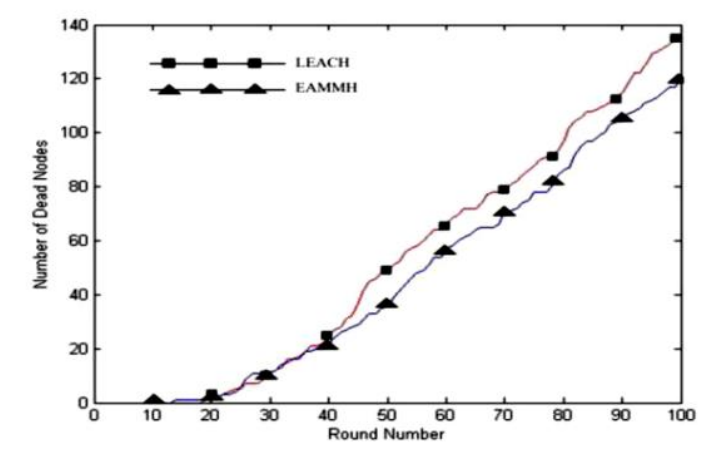

Figure (15):200 nodes

Figures 12,13,14 and 15 represents the comparison of LEACH and EAMMH protocols for the number of dead nodes against the round number elapsed for 50,100,150 and 200 nodes respectively for a cluster head probability of $(0.1)$. In all the figures we can observe that after a total of 100 rounds the number of dead nodes resulting from EAMMH protocol is less than the number of dead nodes resulting from LEACH protocol.

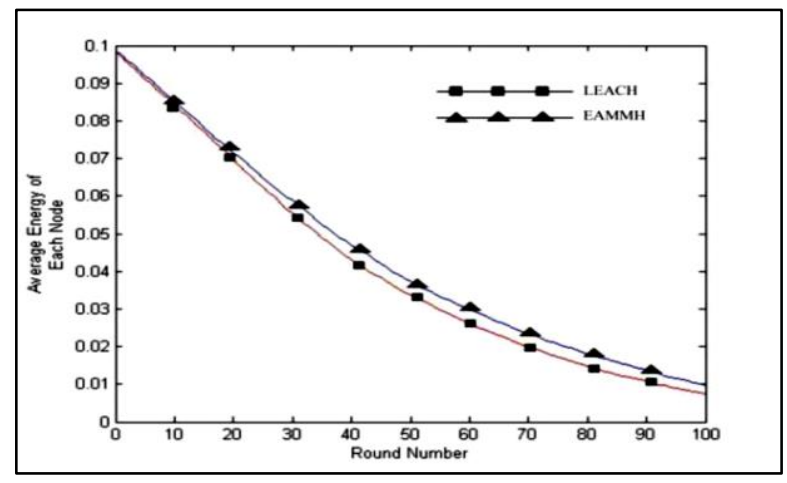

Figure (16):50 nodes

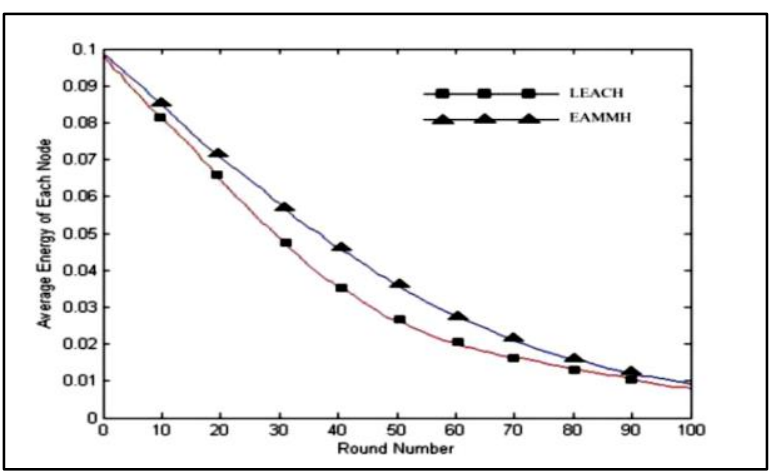

Figure (17):100 nodes

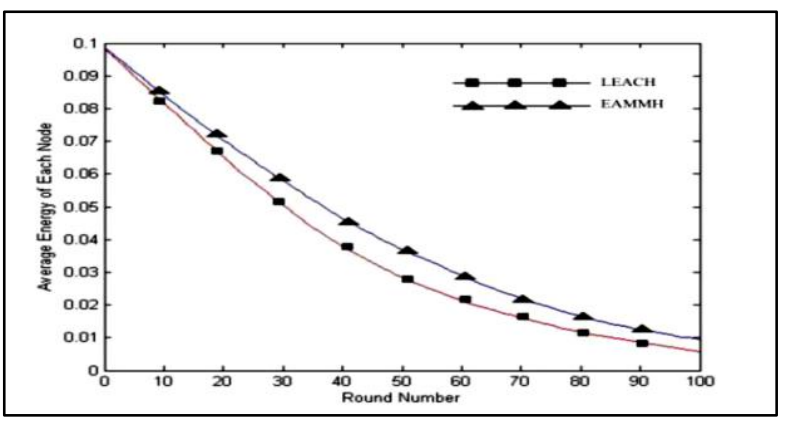

Figure (18):150 nodes

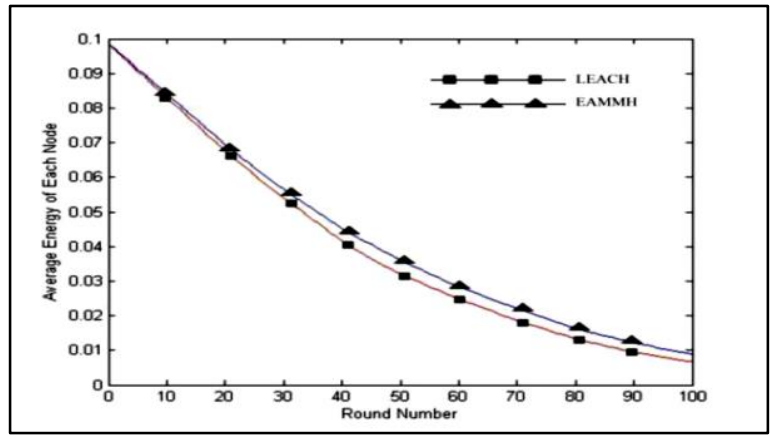

Figure (19):200 nodes

Figures 16,17,18 and 19 represents the average energy of each node as the round progresses for LEACH and EAMMH protocols for the cluster head selection probability of $10 \%$ or 0.1 . We observe from the figures that the average energy of each node using EAMMH protocol after 100 rounds is better in all scenarios of different nodes when compared to LEACH. 


\subsection{Simulation of Protocols at 0.2 probability}

The above set of results represent the simulation of both LEACH and EAMMH protocols at 0.2 probability that is the percentage of total nodes which can become cluster head is $20 \%$ of the total number of nodes.

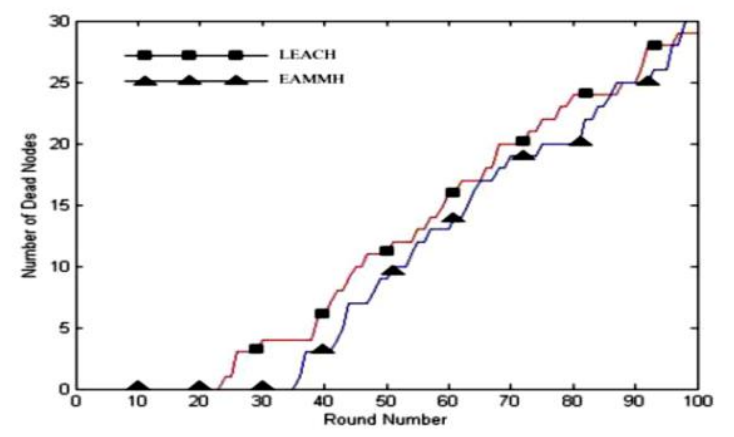

Figure (20):50 nodes

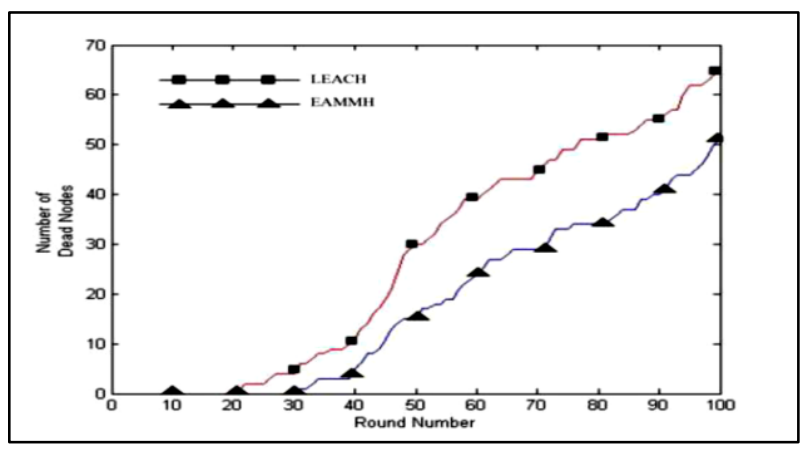

Figure (21):100 nodes

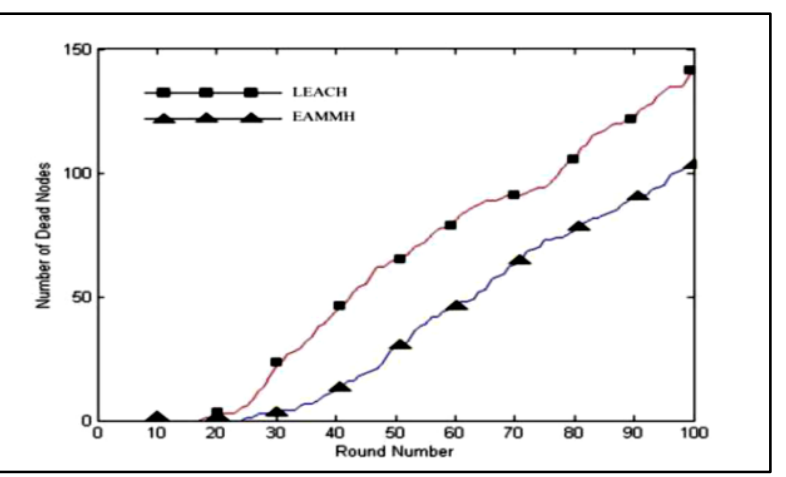

Figure (22):150 nodes

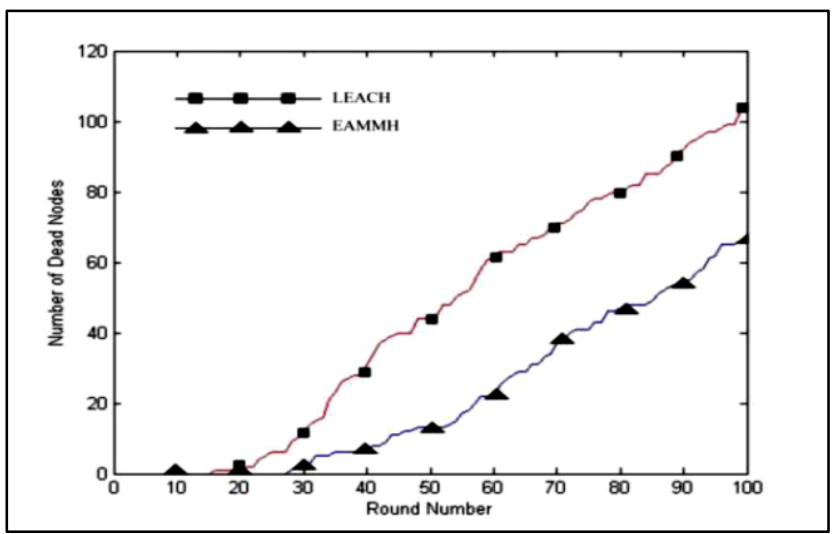

Figure (23):200 nodes

Figures 20,21,22 and 23 represents the comparison of LEACH and EAMMH protocols for the number of dead nodes against the round number elapsed for 50,100,150 and 200 nodes respectively for a cluster head probability of 0.2 . We observe in Figure 20 , with a simulation of a total of 50 nodes that the number of dead nodes after 100 rounds is 29 and 30 respectively for LEACH and EAMMH protocols. LEACH protocol performs slightly better than EAMMH when the number of nodes is 5 , whereas as the number of nodes increases, we observe from Figures 21,22 and 23 that EAMMH outperforms LEACH in all the scenarios.

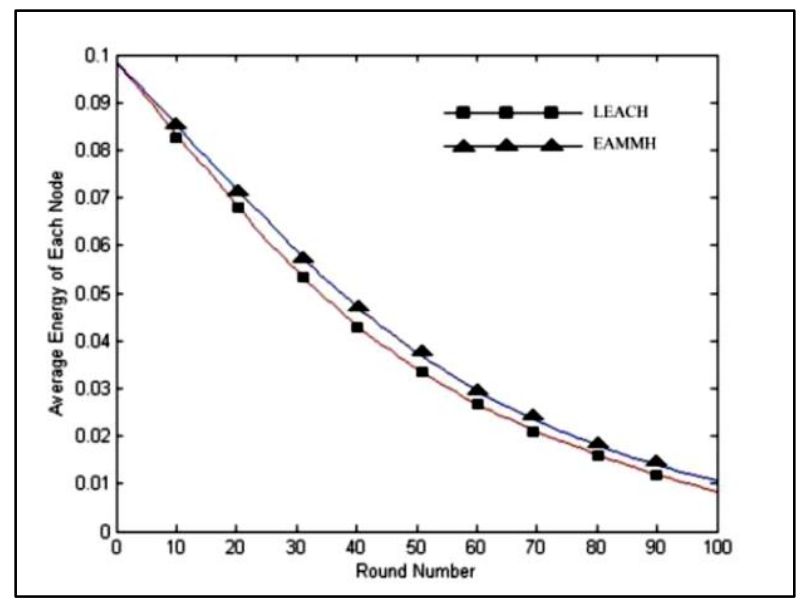

Figure (24):50 nodes 


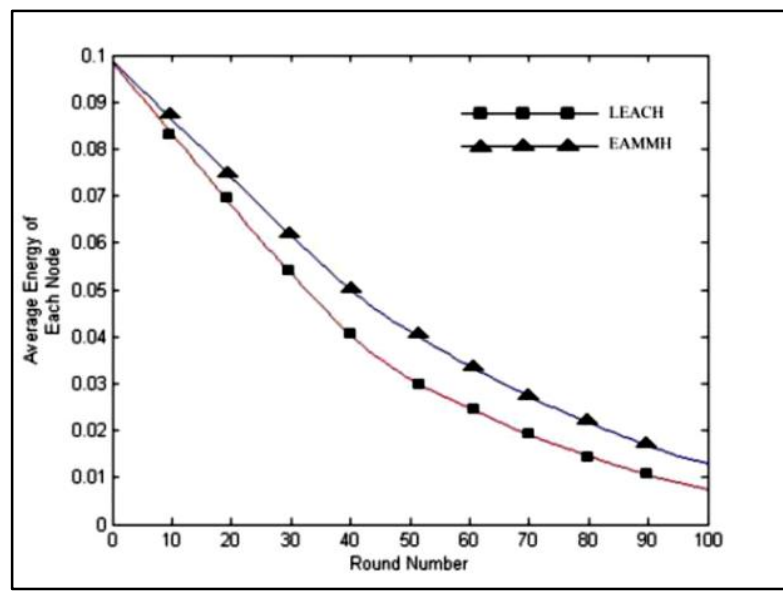

Figure (25):100 nodes

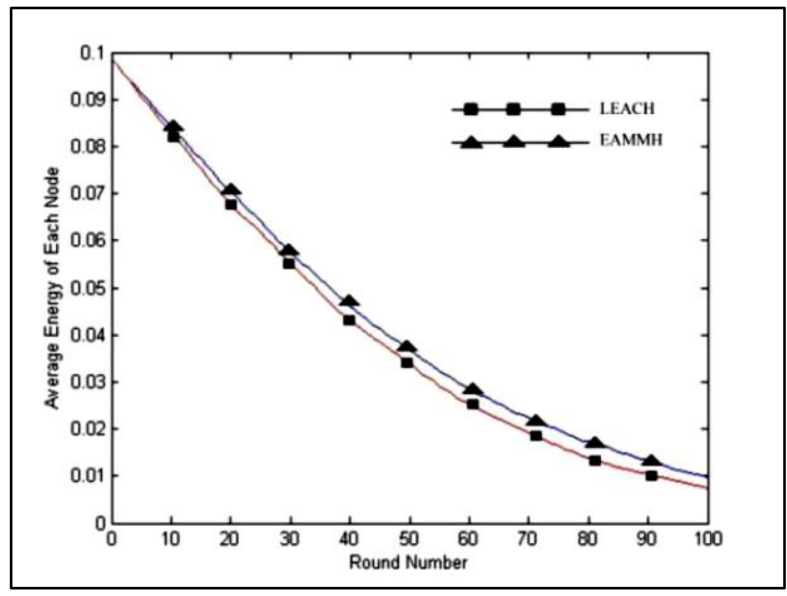

Figure (26):150 nodes

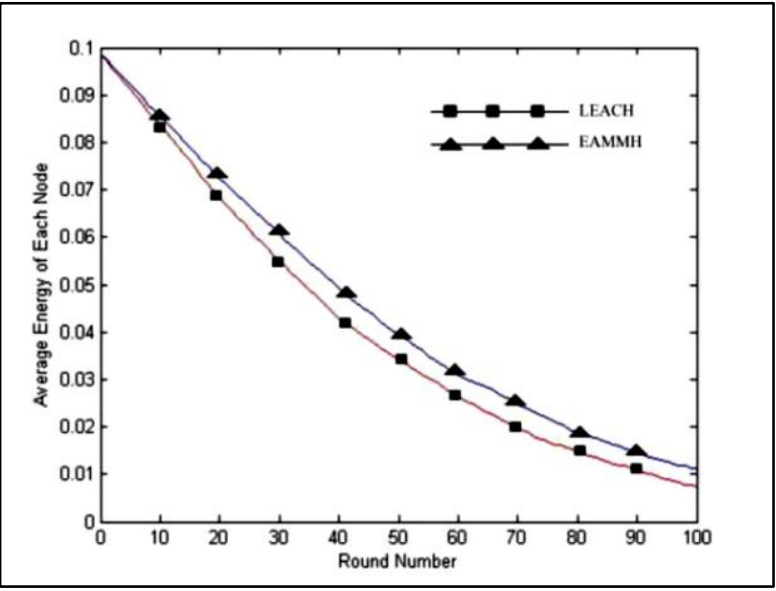

Figure (27):200 nodes
Figures 20,21,22 and 23 represents the average energy of each node as the round progresses for LEACH and EAMMH protocols for the cluster head selection probability of $20 \%$ or 0.2 . From Figure 22, we observe that the average energy of each node curve for both EAMMH and LEACH is very close after 100 rounds, where in EAMMH energy is slightly better than LEACH. From Figures 23,24 and 25 we observe that the energy gap of the curves of EAMMH and LEACH vary significantly with EAMMH outperforming LEACH protocol.

\section{Analyses Of Results}

It is observed from the figures 2 to 25 ,that as time progress LEACH and EAMMH both lose energy as the number of round increases. It is also observed that once a node reaches the value of zero it is no longer functional and is deemed as a dead node. From Figures 4-11 we observe that as the number of nodes increase EAMMH curve for average energy of each node is slightly better. The numbers of dead nodes also get lesser as the number of total nodes increase when compared to LEACH. Therefore for a probability of 0.05 as the number of nodes increases, the better is EAMMH when compared to LEACH. From the Figures 12-27, it is be evident that for each probability level as the number of nodes increase EAMMH is seen to perform better in terms of average energy of each node and the total number of dead nodes. However for a lesser number of total number of nodes, LEACH is found to perform better. From the Figures we observe from most cases that even though EAMMH performs better, the first dead node in most of the operations is by EAMMH. LEACH on the other hand has a delayed time in getting the first dead node but a larger number of nodes run out of energy in a short period of time subsequently. From the Figures, it can also be observed that for a fixed set of nodes, if the probability of election of Cluster Head is increased, then the average energy of each node gap between the curves increases favoring EAMMH. From Figure 7,15 and 23 we observe that LEACH at 0.05 probability is better than EAMMH, while at a probability of 0.1 , EAMMH outperforms LEACH by a factor of $25 \%$ and at 0.2 probability by a factor of around $45 \% /$. The number of dead nodes from Figure 7 for EAMMH and LEACH is at 62 and 68 , from Figure 15, 57 and 62 , from Figure 23, 51 and 62 respectively. From the simulations we observe that the nodes which are far away from the base station are the ones which run out of energy more 
quickly than the rest which are nearer to the Base Station. This is due to the fact that the nodes or the $\mathrm{C} \mathrm{H}$ which are farther from the Base Station have to dissipate large amounts of energy to send the information as they will have to travel longer distances when compared to the ones which are nearer.The reason why EAMMH performs better than LEACH in majority of the scenarios is for the reason that EAMMH consists of a inter cluster routing mechanism which will help make the network survive for a longer time. LEACH on the other hand has a direct hop communication with the Cluster Head and then to the Base Station. Even though LEACH employs Multi-hop mechanisms, EAMMH with the usage of Multi-path and hierarchical routing parameters and techniques with the inclusion of Multihop can perform with much better energy efficiency than LEACH in cases where more number of nodes are involved. In cases when there are a few nodes as an intra-cluster routing mechanism can add to the overhead of the node, LEACH in its simple mode of operation proves to be more energy efficient.

\section{Conclusion}

Wireless Sensor Networks are usually spread over large areas are recently finding applications in many fields. In this regard, there is a requirement of methods which can manage the WSN's in a better way. Wireless Sensor Networks are powered by the limited capacity of batteries. The main challenge in the design of protocols for wireless sensor network is energy efficiency due to the limited amount of energy in the sensor nodes. The ultimate motive behind any routing protocol is to be as energy efficient as possible to keep the network running for a longer period of time. In this paper we have presented clustering as a means to overcome this difficulty of energy efficiency.Detailed description about the working of two protocols, namely LEACH and EAMMH are presented. We have also presented the details about the simulation and the results of it. From the brief analyses of the simulation we have come to a conclusion that LEACH can be preferred in cases of smaller networks where the total number of nodes is less than fifty where it performs slightly better than EAMMH and EAMMH can be chosen in larger networks and also when the heuristic probability of Cluster Head selection is more.

\section{References}

[1] Lan F. Akyildiz, Weilian Su, Yogesh Sankarasubramaniam and Erdal Cayirci, "A
Survey on Sensor Networks", IEEE communications Magazine, August 2002.

[2] Moghaddam N., Zahmati. A and Abolhassani B.," Lifetime Enhancement in WSN's Using Balanced Sensor Allocation to Cluster Heads", IEEE international conferences on signal processing and communications, Dubai, United Arab Emirates, November 2007.

[3] Jennifer Yick, Biswanath Mukherjee, Dipak Ghosal, Wireless sensor network survey, Computer Networks, Volume 52, Issue 12, 22 August 2008.

[4] I.F. Akyildiz, W. Su, Y. Sankarasubramaniam, E. Cayirci, Wireless sensor networks: a survey, Computer Networks, Volume 38, Issue 4, 15 March 2002.

[5] Rajaravivarma, V.; Yi Yang; Teng Yang; , "An overview of Wireless Sensor Network and applications," System Theory, 2003. Proceedings of the $35^{\text {th }}$ Southeastern Symposium on,1618March-2003.

[6] Heinzelman W., Chandrakasan A. and BalakrishnanH.,"Energy-Efficient

Communication Protocol for Wireless Mirosensor Networks", Proceeding of the $33^{\text {rd }}$ Hawii International Conference on System Science, 2000.

[7] Barolli L., Ando H., Xhafa F., Durresi A., Miho R. and Koyama A., "Evaluation of An Intelligent Fuzzy-Based Cluster Head Selection System for WSNs using Different Parameters", International Conference on Advanced Information Networking and Applications,2011.

[8] Mittal N., Singh D., Panghal A. and Chauhan R., "Improved LEACH Communication Protocol for WSN", National Conference on Computational Instrumentation, 2010.

[9] Monica $\mathrm{R}$ Mundada, $\mathrm{V}$ CyrilRaj and $\mathrm{T}$ Bhuvaneswari. "Energy Aware Multi-Hop MultiPath Hierarchical (EAMMH) Routing Protocol for Wireless Sensor Networks" European Journal of Scientific Research, Vol. 88 No 4 October, 2012

[10] Parul Bansal, Poonam Kundu, and Prabhjot Kaur " Comparison of LEACH and PEGASIS Hierarchical Routing Protocols in Wireless Sensor Networks" Int. J. of Recent Trends in Engineering \& Technology, Vol. 11, June 2014 . 